\title{
Do monkeys think in metaphors? Representations of space and time in monkeys and humans
}

\author{
Dustin J. Merritt ${ }^{\mathrm{a}, *}$, Daniel Casasanto ${ }^{\mathrm{b}, \mathrm{c}, \mathrm{d}}$, Elizabeth M. Brannon ${ }^{\mathrm{e}}$ \\ a Center for Cognitive Neuroscience, Duke University, Box 90999, Durham NC 27708, United States \\ ${ }^{\mathrm{b}}$ Max Planck Institute for Psycholinguistics, Nijmegen, NL, The Netherlands \\ ${ }^{\mathrm{C}}$ Donders Institute for Brain, Cognition \& Behaviour, Nijmegen, NL, The Netherlands \\ ${ }^{\mathrm{d}}$ Department of Psychology, The New School for Social Research, New York, NY, United States \\ ${ }^{\mathrm{e}}$ Department of Psychology and Neuroscience E Center for Cognitive Neuroscience, Duke University, Box 90999, Durham NC 27708, United States
}

\section{A R T I C L E I N F O}

\section{Article history:}

Received 9 October 2009

Revised 5 July 2010

Accepted 11 August 2010

\section{Keywords:}

Metaphor

Time

Space

Monkeys

Embodied cognition

\begin{abstract}
A B S T R A C T
Research on the relationship between the representation of space and time has produced two contrasting proposals. ATOM posits that space and time are represented via a common magnitude system, suggesting a symmetrical relationship between space and time. According to metaphor theory, however, representations of time depend on representations of space asymmetrically. Previous findings in humans have supported metaphor theory. Here, we investigate the relationship between time and space in a nonverbal species, by testing whether non-human primates show space-time interactions consistent with metaphor theory or with ATOM. We tested two rhesus monkeys and 16 adult humans in a nonverbal task that assessed the influence of an irrelevant dimension (time or space) on a relevant dimension (space or time). In humans, spatial extent had a large effect on time judgments whereas time had a small effect on spatial judgments. In monkeys, both spatial and temporal manipulations showed large bi-directional effects on judgments. In contrast to humans, spatial manipulations in monkeys did not produce a larger effect on temporal judgments than the reverse. Thus, consistent with previous findings, human adults showed asymmetrical space-time interactions that were predicted by metaphor theory. In contrast, monkeys showed patterns that were more consistent with ATOM.
\end{abstract}

(c) 2010 Elsevier B.V. All rights reserved.

\section{Introduction}

The question of how representations of time, space, and number interact has been the focus of much research. Critical behavioral and neurological linkages have been found between number and time (Gallistel \& Gelman, 2000; Meck \& Church, 1983; Oliveri et al., 2008), space and time (Basso, Nichelli, Frassinetti \& diPellegrino, 1996; Boroditsky, 2000; Casasanto \& Boroditsky, 2008; Delong, 1981; Sarrazin, Giraudo, Pailhous, \& Bootsma, 2004; Srinivasan \& Carey, 2010; Vicario et al., 2008), and space and number

\footnotetext{
* Corresponding author. Tel.: +1 919668 0437; fax: +1 9196810815 . E-mail address: djm20@duke.edu (D.J. Merritt).
}

(Dehaene, Bossini, \& Giraux, 1993; Hubbard, Piazza, Pinel, \& Dehaene, 2005). Walsh (2003a) synthesized these findings into a theory of magnitude (ATOM) which asserts that time, space, and number are all processed by a common analog magnitude system, and depend on common neural structures (see also, Cantlon, Platt, \& Brannon, 2009; Feigenson, 2007; Meck \& Church, 1983). ATOM predicts that the co-occurrence of time, space, and number should produce priming or interference across dimensions. But because these dimensions are represented by a common metric, ATOM also implies that priming or interference across dimensions should be roughly symmetrical (Casasanto, Fotakopoulou, \& Boroditsky, 2010). For example, variations in space should influence judgments of time to about the same degree that variations in time influence 
judgments of space. A priori, ATOM provides no reason to posit that representations in one of these domains should have any special dependence on representations in the other.

According to metaphor theory, however, the relationship between time and space is asymmetrical. Representations of time depend on representations of space, more than vice versa (Boroditsky, 2000; Bottini \& Casasanto, 2010; Casasanto \& Boroditsky, 2008; Casasanto, Fotakopoulou, \& Boroditsky, 2010; Clark, 1973; Lakoff \& Johnson, 1980). Because we can directly perceive movement through space, but can only imagine movement through time, we use spatial representations to help us think about time. Evidence for this view can be found in the distribution of space-time metaphors in language. Spatial metaphors are used to describe temporal relationships more frequently than temporal metaphors are used to describe spatial relationships (Lakoff \& Johnson, 1980). For example, it is common to say that a meeting was moved forward two days, that the past is behind us, that we're taking a short break or a long vacation. Although some temporal expressions can be used to describe relationships in space (e.g. we are five minutes away from the movie theater), they are less common.

An important question is whether these spatial metaphors in language capture the way people think about relationships in time, or just the way they communicate about them. As Murphy $(1996,1997)$ explains, relying on purely linguistic analyses can lead to logical circularity when the metaphors themselves are offered as evidence for the metaphorical representation of abstract concepts. Thus, independent psychological evidence is needed. To avoid confusion, it is important to distinguish between metaphors in language (linguistic metaphors) and the non-linguistic structures upon which linguistic metaphors are theoretically based (mental metaphors) (e.g. Casasanto, 2009a). There is no doubt that people use linguistic metaphors to help them talk about time. Do they also use mental metaphors from space to help them think about the more abstract domain of time?

To address this question, rather than studying static patterns in language, some of the first experimental tests examined how people process language about space and time (Boroditsky, 2000). In English, two types of spatial schemas are used to organize temporal events (Clark, 1973). One type is an ego-moving schema in which the individual is conceptualized as moving through time (e.g. we are approaching Christmas). The other type is a timemoving schema in which time is moving toward the stationary individual (e.g. Christmas is approaching). In Boroditsky's experiment, participants answered a series of spatial priming questions that were composed of a sentence and a picture, after which, they were asked an ambiguous question about time (Next Wednesday's meeting has been moved forward two days. What day is the meeting?). Boroditsky found that participants' responses to the ambiguous temporal question were consistent with the spatial primes. When the spatial primes were time moving, participants were more likely to answer Monday, and when spatial primes were ego-moving, participants were more likely to answer Friday.
If people really think about time by activating spatial representations (more than vice versa), then asymmetric space-time interactions should extend beyond the domain of language. To test this proposal, Casasanto and Boroditsky (2008) conducted a series of psychophysical tasks with non-linguistic stimuli and responses. In each task, Englishspeaking adults viewed lines or dots on a computer screen and reproduced either the distance they covered or the amount of time they remained on the screen, using mouse clicks to indicate the beginning and end of each spatial or temporal interval. All six experiments showed that the longer a stimulus extended in space, the longer participants judged that it lasted in time. By contrast, the temporal extent of stimuli did not influence judgments of their spatial extent. Control analyses showed that this difference in cross-dimensional interference was not due to differences in how accurately participants could estimate distance and duration, per se. These experiments reveal that the asymmetric space-time relationship found in language is also found for non-linguistic mental representations of space and time. Results support metaphor theory, but are inconsistent with the most straightforward predictions derived from ATOM.

But perhaps representations of space and time start out ATOMic in the course of cognitive development, and only later become metaphoric? To investigate this possibility, Casasanto, Fotakopoulou, and Boroditsky (2010) conducted a series of space-time interference experiments in kindergarten and elementary school aged children. Children were asked to judge either the spatial or the temporal dimension of each stimulus (i.e., pairs of animals 'racing' along parallel paths for different distances and durations). Children could ignore irrelevant temporal information in the stimuli when making judgments about space, but they had much greater difficulty ignoring irrelevant spatial information when making judgments about time. This difference in cross-dimensional interference remained significant even when children's performance judging time per se and space per se was equated. Furthermore, the asymmetric influence of space on time did not vary with the age of the children tested (4-6 y.o. vs. 9-10 y.o.) Although it is not known whether this relationship extends to even younger children, these results show that space and time are already asymmetrically linked in children's minds by about age 5 , several years before they develop adult-like conceptions of duration (Piaget, 1927/1969).

Data from both human adults and children show an asymmetric relationship between space and time, consistent with metaphor theory and inconsistent with ATOM. Yet, much of the data Walsh (2003a) offered as evidence for ATOM came from non-humans, monkeys in particular (e.g., Leon \& Shadlen, 2003; Nieder, Freedman, \& Miller, 2002; Onoe et al., 2001; Sawamura, Shima, \& Tanji, 2002; Stein, 1989; Walsh, 2003b; Wilson, Scalaidhe, \& Goldman-Rakic, 1993). The present study investigated whether the space-time asymmetry found in humans is also found in non-human primates. We tested two rhesus monkeys using an interval bisection procedure (e.g., Meck \& Church, 1983). Participants made judgments about one dimension (e.g. time) while another irrelevant dimension varied (e.g. space). Sixteen adult humans were also tested. The goal 
was to determine the degree to which time and space influenced judgments of one another, and to compare the cross-dimensional interference effects between humans and monkeys.

We identified three possible outcomes, for both species. If representations of space and time are independent, then we would expect no interference between the two dimensions. Variations in time should not influence judgments of space, and neither should variations in space influence judgments of time. On another possibility, consistent with ATOM, if time and space do interfere with one another, they should do so symmetrically. Time should affect space judgments to about the same degree that space affects time judgments. Finally, consistent with metaphor theory, if representations of time are asymmetrically dependent on representations of space, then space should affect time judgments more than time affects space judgments.

\section{Experiment 1}

The goal of Experiment 1 was to replicate Casasanto and Boroditsky's (2008) finding that the spatial length of a line influences duration judgments more than the reverse with adult humans, using a procedure that would be suitable for later use with non-human primates.

\subsection{Method}

\subsubsection{Subjects and apparatus}

Participants were 13 undergraduate students from Duke University who participated in exchange for payment. Three of the original 16 participants were not included in the final sample because they failed to meet a performance criterion in the initial training phase of the study. Stimuli were presented on a 17-in. touch-screen. A custom built program written in Realbasic presented the stimuli and registered responses.

\subsubsection{Procedure}

The participants learned to classify lines on the basis of both duration and spatial extent, with each being trained separately. During duration anchor training, participants were presented with a short (1 s) or a long (4s) duration and were required to choose one symbol if the duration was short and another if the duration was long. During spatial extent anchor training, participants chose one symbol for a short line $(6 \mathrm{~cm})$ and another for a long line $(24 \mathrm{~cm})$.

Participants were not given explicit verbal instructions about how to solve the task. This was done to parallel the necessary lack of verbal instructions in the subsequent monkey experiments. They were informed that the task may seem confusing at first, but through trial and error, they would quickly learn the rules of the game. After being trained on a particular dimension, participants were then given a bisection test on the same dimension before being trained and tested on the other dimension. During bisection testing, participants were given values that were intermediate between the two anchors in order to determine the point of subjective equality (PSE), or the value that was equally likely to be classified as long or short. The order in which the dimensions were trained and tested was counter-balanced across participants.

Training accuracy was assessed for each block of 10 trials, and anchor training continued until the participant reached a criterion performance of 8 out of 10 correct trials within a block, or until they completed 60 trials. All responses were made by physically touching stimuli presented on a touch sensitive monitor. Throughout the experiment, participants initiated each trial by pressing a white square in the center of the screen. A stimulus line then appeared in the center of the screen, with the two response symbols in the lower portion of the screen. The lines appeared in stationary positions with respect to the top and bottom of the screen, but line position varied randomly between 4.5 and $6.5 \mathrm{~cm}$ from the leftmost edge of the screen. Note that a previous study showed that performance was no different when lines dynamically expanded across the screen compared to when they were static (Casasanto \& Boroditsky, 2008, Experiment 6).

The background screen color was orange during duration trials and green during spatial length trials (Fig. 1). Touches to the correct response symbol produced a red screen, a reward tone, and the word "CORRECT!" flashed across the center of the screen for $3 \mathrm{~s}$. Touches to the incorrect response symbol produced a black screen, a tone, and the word "INCORRECT!" flashed across the screen for $3 \mathrm{~s}$.

2.1.2.1. Spatial length anchor training. During spatial length training, the stimulus line was either 6 or $24 \mathrm{~cm}$. The line remained on the screen for $2 \mathrm{~s}$, which was the geometric mean of the two anchors used in duration anchor training. ${ }^{1}$ The participant then chose between two response symbols (a dark blue circle and a red pentagon) (see Fig. 1). One symbol served as the correct response for the short line length, and the other as the correct response for the long line length. The left-right positions of the response symbols were randomized. Anchor training continued until the participant reached an accuracy of $80 \%$ correct within a particular 10 trial block, or 60 trials had elapsed.

2.1.2.2. Spatial length bisection testing. Once each participant reached criterion with the anchor values, a bisection test was given. During bisection testing, the participants were given 120 trials, with $50 \%$ of the trials consisting of the anchor values they received during training, and the other $50 \%$ consisting of five intermediate values. The five intermediate values were equally spaced on a logarithmic scale between the two anchors (7.6, 9.5, 12, 15.1, and $19 \mathrm{~cm}$ ). Anchor values were reinforced as described for training sessions, but intermediate values were non-differentially reinforced. In all experiments, Weibull fits on the psychophysical functions were used to obtain the PSE's.

\footnotetext{
1 Meck and Church (1983) used a similar procedure to test how number and time were represented following deliberate confounding of the two dimensions. In our case, we trained each dimension separately, and later introduced variations in one dimension in order to determine whether it biased judgments of the other.
} 
Line Length Trial
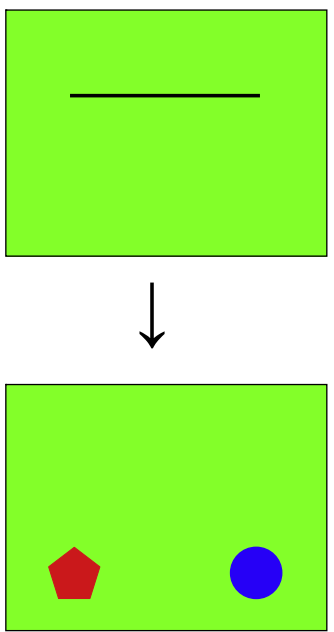

Fig. 1. An example anchor training trial for line-length (left) and duration (right). The background color of the screen indicated whether to judge line-length (green background) or duration (orange background). In each case, a line was presented for a period of time, and then disappeared. Judgments were made by selecting the symbol that corresponded to either the "short" or "long" anchor (line-length or duration). (For interpretation of the references to color in this figure legend, the reader is referred to the web version of this article.)

2.1.2.3. Duration anchor training. Duration anchor training followed the same protocol as anchor training for spatial length. However, instead of judging the spatial length of the line, the participants judged the duration that the line was presented on the screen. The short anchor value was $1 \mathrm{~s}$ and the long anchor value was $4 \mathrm{~s}$ in duration. The spatial length of the line was equal to the geometric mean of the two training anchors in the spatial length training condition $(12 \mathrm{~cm})$. The choice symbols were a black triangle and a cyan plus sign (see Fig. 1). The left-right positions of the response symbols were randomized. Reward contingencies, number of trials per session, and accuracy criterion were the same as they were during spatial length anchor training.

2.1.2.4. Duration bisection testing. Bisection testing for duration followed the same protocol as the bisection testing for spatial length, Given that magnitude judgments for several prothetic dimensions (including space and time) follow Weber's Law, we again used logarithmically spaced intervals for the five intermediate values for duration bisection testing (1260, 1587, 2000, 2520, and $3175 \mathrm{~ms}$ ). This was to ensure that subjective distances between intervals were equivalent both within and between dimensions.

2.1.2.5. Cross-dimensional testing. This phase was similar to bisection testing in that participants made judgments about anchors and intermediate values. However, there were three primary differences. First, participants were required to judge spatial extent on some trials, and duration on other trials, and the two trial-types occurred in random order. Second, there were no cues to inform the participants about whether they were to judge the duration or spatial extent of a line until after the stimulus line was removed from the screen. The stimulus lines were presented on a light gray screen and only after the line disappeared, and the judgment symbols appeared, did the screen color change to green or orange. This was done in order to force participants to process both space and time simultaneously. Third, the irrelevant dimension was no longer held constant at the geometric mean (see Fig. 2). Instead, the irrelevant dimension held one of three values; short, medium, or long. For example, when the participants made a line-length judgment, all seven spatial extents were tested (two anchors and five intermediate values). However, the line's duration could be one of the two duration anchor values (short or long) or the geometric mean of those values (medium). Thus, for each condition (time and space) there were 21 different trial-types $(3 \times 7)$. Participants were given a total of 392 test trials; $50 \%$ of those trials were reinforced anchor trials, with the other $50 \%$ consisted of non-differentially reinforced test trials.

\subsection{Results}

\subsubsection{Bisection testing}

The participants showed the expected sigmoid-shaped bisection function during both spatial length and duration bisection testing. The PSE for spatial length was $10.41 \mathrm{~cm}$ (geometric mean $=12 \mathrm{~cm}$ ), and the PSE for duration was $1778.6 \mathrm{~ms}$ (geometric mean $=2000 \mathrm{~ms}$ ). Calculation of the Weber Fraction (WF), a measure that indicates the minimum change needed to detect a difference between two stimuli, revealed that sensitivities were similar for
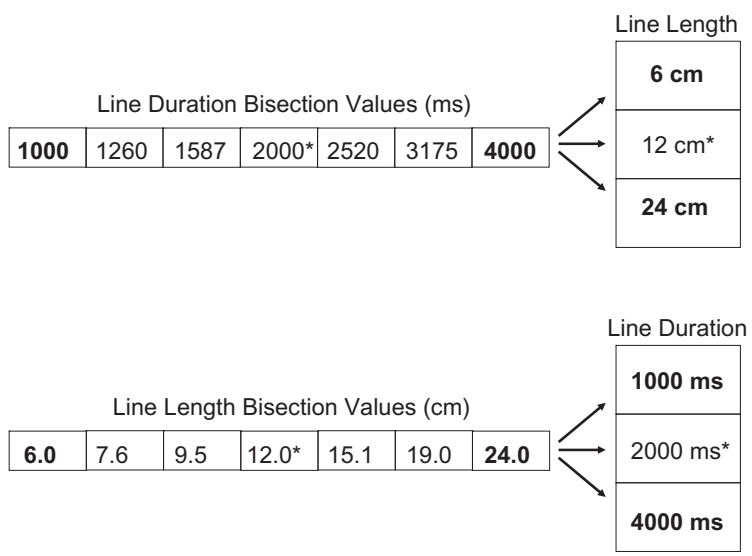

Fig. 2. Stimulus values for temporal and spatial cross-dimensional bisection. In the duration bisection test, participants judged seven durations (two anchors and five intermediate values) as long or short while the spatial extent of the lines were orthogonally varied between short, medium, and long values to produce 21 different duration (seven values $) \times$ spatial length (three values) stimuli. Note that the three spatial lengths used were the short and long anchors and the geometric mean of these values. In the spatial bisection test, participants judged seven line lengths as long or short while the duration of the lines were orthogonally varied between short, medium, and long values to produce 21 different spatial (seven values) $\times$ duration length (three values) stimuli. Note that the three durations used were the short and long anchors and the geometric mean of these values. Short and long anchors for both dimensions are indicated via bolded text above, and the geometric mean is indicated with an asterisk. 

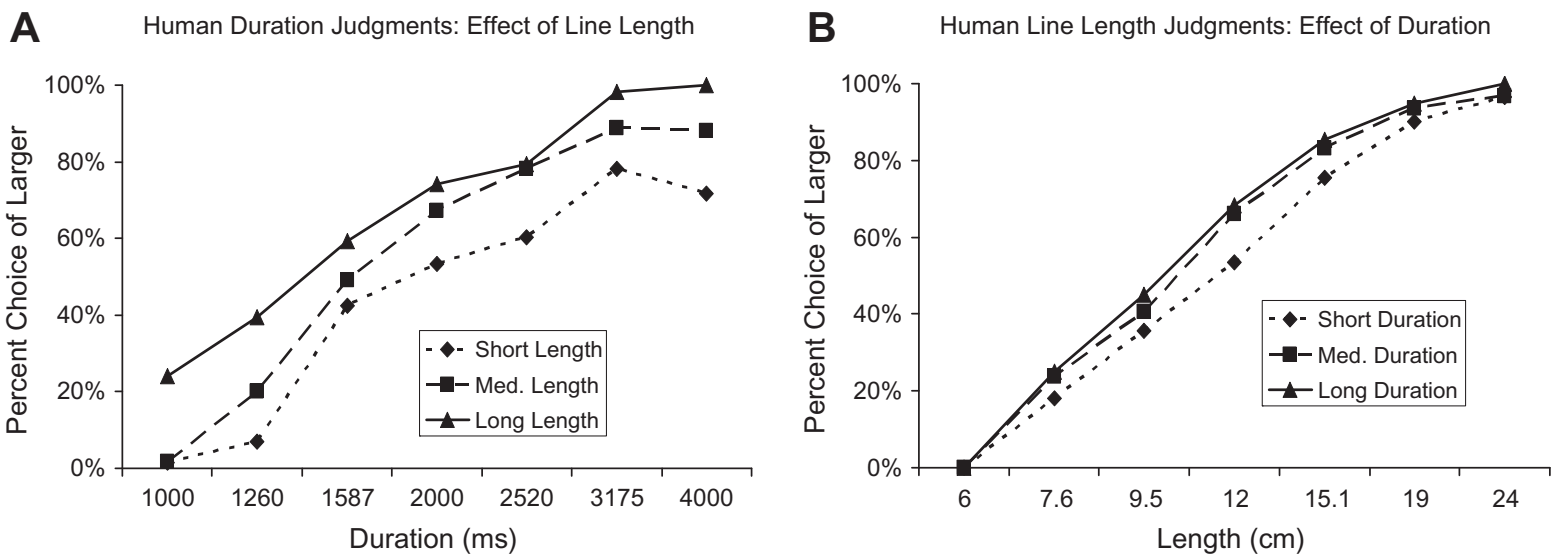

Fig. 3. (A) The human bisection functions for judgments of duration when crossed with short $(6 \mathrm{~cm})$, medium (12 cm), and long ( $24 \mathrm{~cm})$ spatial lengths. (B) The human bisection functions for judgments of spatial length when crossed with short (1000 ms), medium (2000 ms), and long (4000 ms) durations.

both duration, $\mathrm{WF}=0.24,95 \%$ confidence intervals (C.I.) $0.20-0.28$, and line-length $(\mathrm{WF}=0.22$, 95\% C.I. 0.19-0.25) judgments.

\subsubsection{Cross-dimensional testing}

In order to examine the effects of the irrelevant dimension on the judgments of the relevant dimension, we conducted a mixed-effects logistic regression (with subject as a random effect) for time and space judgments using the seven values of the judged dimension and the three values of the irrelevant dimension as predictors of "long" or "short" responses. We also tested for asymmetry using a mixed-effects logistic regression with subject as a random effect, and the seven values of the judged dimension, the three values of the irrelevant dimension, the judgment condition (space or time), and the interaction between the judgment condition and the three values of the irrelevant dimension as predictors. A Wald chi-square was used to assess the unique contribution of each predictor.

Our results were consistent with prior work by Casasanto and Boroditsky (2008) in that bias was asymmetrical, with space influencing time judgments more than time influenced space judgments. As shown in Fig. 3, when making judgments of duration crossed with small, medium, and large spatial lengths, the PSE values were $1972 \mathrm{~ms}, 1651.5 \mathrm{~ms}$, and $1447.5 \mathrm{~ms}$ (Fig. 3A). When making judgments of spatial length crossed with small, medium, and large duration values, the PSEs were $11.3 \mathrm{~cm}$, $10.19 \mathrm{~cm}$, and 9.86 respectively (Fig. 3B). Analyses revealed that time biased judgments of space, and vice versa [Time on space, $\chi 2(1, N=1269)=10.76, p<0.01$, odds ratio $=$ 1.48 , 95\% C.I. 1.13-1.83; Space on time, $\chi^{2}(1, N=1268)=$ $68.00, p<0.001$, odds ratio $=2.25$, 95\% C.I. $1.82-2.68$ ]. Importantly, similar to previous findings with humans, spatial length influenced judgments of duration to a greater degree than duration influenced judgments of spatial length as indicated by the interaction between the irrelevant dimension and the judgment condition in the model, $\chi^{2}(1, N=2537)=19.66, p<0.001$, odds ratio $=1.83(95 \%$ C.I. 1.34-2.32).

\section{Experiment 2a}

The goal of Experiments 2a and 2b was to use the bisection paradigm developed in Experiment 1 to assess whether rhesus monkeys, like human adults, show asymmetry between spatial length and duration. The method of Experiment 2a differed slightly from that of Experiment 1 during cross-dimensional tests in that a cue was given during the line presentation to indicate whether the monkey should attend to line-length or duration. This was done because we were concerned that the monkeys would not understand the task without such a cue.

\subsection{Method}

\subsubsection{Subjects and apparatus}

The subjects were two 5-year old female rhesus macaques (Macaca mulatta), Curry and Bales. Both monkeys were born in captivity and were experimentally naïve prior to this experiment. Subjects were tested in sound attenuated rooms while seated in Plexiglass primate chairs fitted with a juice delivery system. The touch-screen and experimental software were the same as that used with humans.

\subsubsection{Procedure}

Prior to the experiment, both monkeys were shaped to touch a stimulus on the touch-screen to obtain a juice reward. After learning to use the touch-screen, the monkeys were trained to classify lines based on their duration or spatial extent. Curry was trained to classify the duration anchors first and Bales was trained to classify the spatial anchors first. The durations and line lengths were identical to that described in Experiment 1. After reaching a performance criterion of $80 \%$ on the anchor values for two consecutive sessions, monkeys were given the same bisection test described in Experiment 1. After a bisection function was obtained for each monkey on the first condition (duration or line length) each monkey was then trained to classify anchors for the other condition - after which, a corresponding bisection function was obtained. 
Like the human participants in Experiment 1, monkeys were required to initiate each trial by pressing a white square in the center of the screen. The stimulus line then appeared in the center of the screen, with the two response symbols in the lower portion of the screen. The background color of the screen was orange during duration trials and green during spatial length trials (Fig. 1). Correct responses produced a $1.25 \mathrm{ml}$ juice reward with positive auditory feedback, followed by a $3 \mathrm{~s}$ inter-trial interval (ITI). Incorrect responses produced a $1 \mathrm{~s}$ time-out (in addition to the $3 \mathrm{~s} \mathrm{ITI),} \mathrm{negative} \mathrm{auditory} \mathrm{feedback,} \mathrm{and} \mathrm{were}$ not rewarded with juice. During both training and testing, sessions consisted of 150 trials.

3.1.2.1. Spatial length anchor training. Stimuli and trial structure was identical to that described in Experiment 1 for human subjects (Fig. 1). The only difference was that anchor training continued until the monkey had successfully completed two consecutive sessions at $80 \%$ correct.

3.1.2.2. Spatial length bisection testing. During bisection testing, the monkeys were presented with the anchor values they received during training on $70 \%$ of the trials, and with the same five intermediate values described in Experiment 1 on $30 \%$ of the trials. Anchor values were reinforced as described for training sessions. Intermediate values were non-differentially reinforced and juice was delivered regardless of whether the stimulus was classified as short or long. Twelve test sessions were completed.

3.1.2.3. Duration anchor training. Stimuli and trial structure was identical to that described in Experiment 1 for duration anchor training for human participants.

Reward contingencies, number of trials per session, and accuracy criterion were the same as they were during spatial length anchor training.

3.1.2.4. Duration bisection testing. Bisection testing for duration followed the same protocol as described above for line-length bisection. The values of the intermediate durations were as described for human subjects in Experiment 1.

3.1.2.5. Randomized anchor training. An additional step was taken with monkeys that was not used in Experiment 1 with humans. Once each monkey had completed anchor training and bisection testing on each dimension (counter-balanced), monkeys were re-trained on anchor values in which spatial length and duration trials were randomly intermixed. Thus monkeys were required to use the background color and symbol identity to determine whether they should judge the stimulus line's duration or spatial extent. Trials were initially given in blocks of 10 trials for each condition, (e.g. 10 duration trials followed by 10 spatial length trials). Once an $80 \%$ accuracy criterion was met for two consecutive sessions, blocks were reduced to five trials, and, once criterion was met again, duration and spatial length trials were randomly intermixed. When the monkeys reached the $80 \%$ accuracy criterion for two consecutive sessions of randomized trials, the monkeys were then moved onto the cross-dimensional testing phase.
3.1.2.6. Cross-dimensional testing. This phase was as described for human participants in Experiment 1. Each monkey was given 162 trials per session for a total of 28 sessions.

\subsection{Results and discussion}

Monkeys learned to classify anchor values for each condition and showed similar sensitivity for duration and linelength judgments. Bales reached criterion performance after 22 sessions of anchor training in the spatial length condition, and after 21 sessions of training in the duration condition. Curry reached criterion performance after 47 sessions of training in the duration condition, and after 39 sessions of training in the spatial length condition.

\subsubsection{Spatial length and duration bisection testing}

The monkeys showed the expected sigmoid-shaped bisection function for line-length and duration (Fig. 4). The PSE value for duration testing was $2298 \mathrm{~ms}$ (Fig. 4A), and for spatial length testing, the PSE was $12.2 \mathrm{~cm}$ (Fig. 4B). Calculation of Weber fractions (WF) revealed that discrimination sensitivities were similar across both duration $(\mathrm{WF}=0.24,95 \%$ C.I. $0.18-0.30)$ and line-length $(\mathrm{WF}=0.19,95 \%$ C.I. $0.12-0.26)$.

\subsubsection{Cross-dimensional testing}

In order to examine the effects of the irrelevant dimension on the judgments of the relevant dimension, we again used a mixed-effects logistic regression analysis using the same parameters described in Experiment 1. When making judgments of duration crossed with small, medium, and large spatial lengths the PSE's were $2592.2 \mathrm{~ms}$, $1892.3 \mathrm{~ms}$, and $1200 \mathrm{~ms}$ respectively (Fig. 5A). Similarly, when making judgments of spatial length crossed with small, medium, and large durations, the PSE's were $16.5 \mathrm{~cm}, 10.18 \mathrm{~cm}$, and 6.07 values respectively (Fig. 5B).

For both judged dimensions, the irrelevant dimension significantly influenced judgments of the relevant dimension [space on time, $\chi^{2}(1, N=1176)=126.70, p<0.001$, odds ratio $=2.92,95 \%$ C.I. 2.38-3.46; time on space, $\chi^{2}(1, N=1176)=152.39, p<0.001$, odds ratio $=3.00,95 \%$ C.I. 2.48-3.52]. However, unlike the patterns previously found in humans, the monkeys did not show asymmetries in the degree to which space and time influenced one another $\chi^{2}(1, N=2352)=2.23, p=0.14$, odds ratio $=1.20$, 95\% C.I. 0.91-1.48. It should be noted that while the monkeys' asymmetry did approach statistical significance, the pattern of influence was opposite that found in the human participants, with time influencing space to a slightly larger degree than the reverse. Furthermore, as shown by the odds ratios, the effect of time on space was relatively small and the effect of space on time was large for humans, whereas the effects were large in both directions for the monkeys.

\section{Experiment 2B}

In the previous experiment, the background color during stimulus presentation informed the monkeys as to 

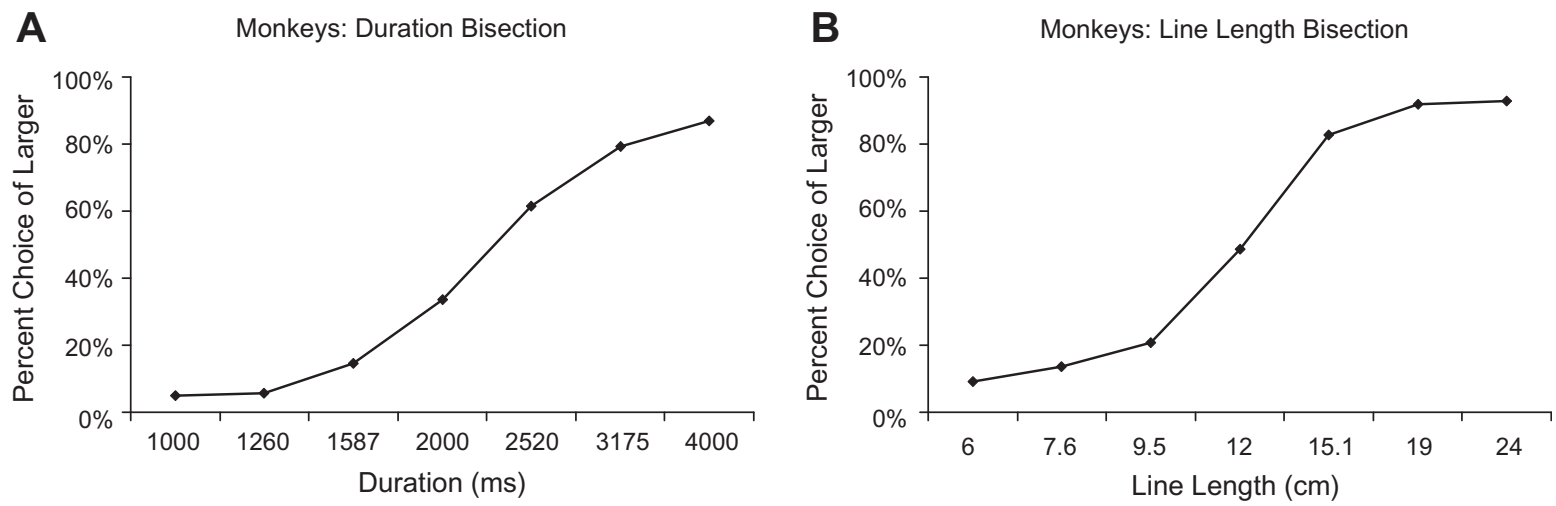

Fig. 4. The monkey bisection functions for duration $(W F=0.24)$ and spatial length $(W F=0.19)$ following anchor training.

A Monkey Duration Judgments: Effect of Line Length

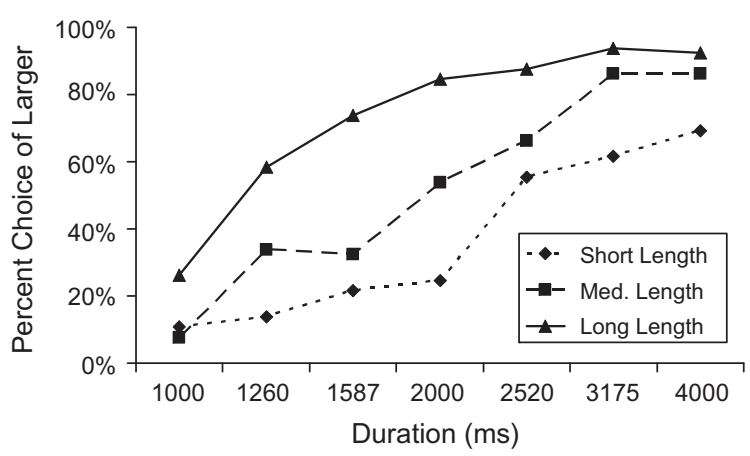

B Monkey Line Length Judgments: Effect of Duration

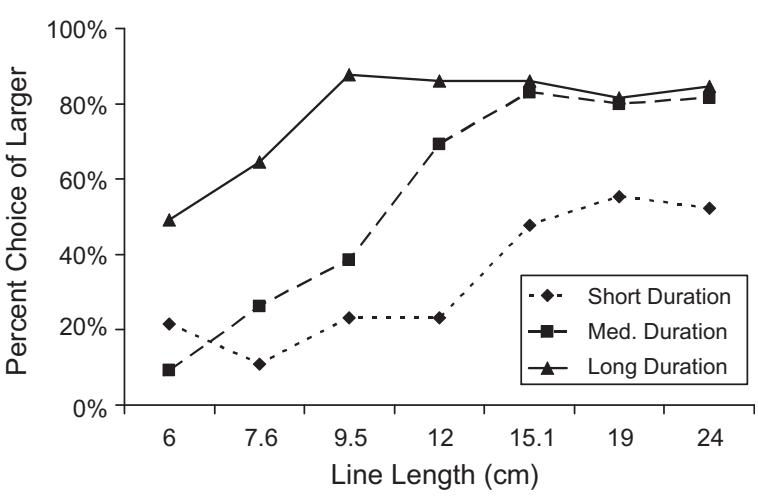

Fig. 5. (A) The bisection functions (in monkeys) for judgments of duration when crossed with short $(6 \mathrm{~cm})$, medium (12 cm), and long (24 cm) spatial lengths. (B) The bisection functions for judgments of spatial length when crossed with short (1000 ms), medium (2000 ms), and long (4000 ms) durations. When the line was shown, the background color of the screen indicated whether to judge line-length (green) or duration (orange). (For interpretation of the references to color in this figure legend, the reader is referred to the web version of this article.)

whether they should attend to the duration or the spatial extent of a line. Knowing the relevant dimension while processing the stimulus may have allowed the monkeys to partially ignore the irrelevant dimension, thereby reducing any asymmetrical effects. To address this, we next tested monkeys in a design more analogous to that used with humans in Experiment 1, where there was no background color cue until the stimulus line disappeared, and the judgment symbols appeared. Under these conditions, the monkeys did not know whether they were supposed to make a judgment of spatial length or a judgment of duration until after they had finished viewing the stimulus. The purpose was to force the monkeys to process both space and time dimensions simultaneously.

\subsection{Method}

\subsubsection{Subjects and apparatus}

The monkeys and apparatus were the same as those in Experiment 2a.

\subsubsection{Procedure}

Both monkeys were given two sessions with anchor values only. This was followed by 12 cross-dimensional testing sessions. These sessions differed from Experiment 2a only in that the stimulus lines were presented on a gray (rather than a green or orange) background. The color cue to make a temporal or line-length judgment appeared only after the stimulus line had disappeared making it necessary for monkeys to represent both the temporal and spatial extent of all stimulus lines.

\subsection{Results and discussion}

The delay of the space vs time cue had little effect on monkeys' performance. Both monkeys showed large bidirectional effects of space and time. When making judgments of duration, the PSE values were $2223.3 \mathrm{~ms}$, $1814 \mathrm{~ms}$, and $1064.5 \mathrm{~ms}$ (Fig. 6A) when crossed with small, medium, and large spatial length values respectively, $\chi^{2}(1, N=1174)=126.37, p<0.001$, odds ratio $=3.06,95 \%$ C.I. 2.46-3.66. Similarly, when making judgments of spatial 

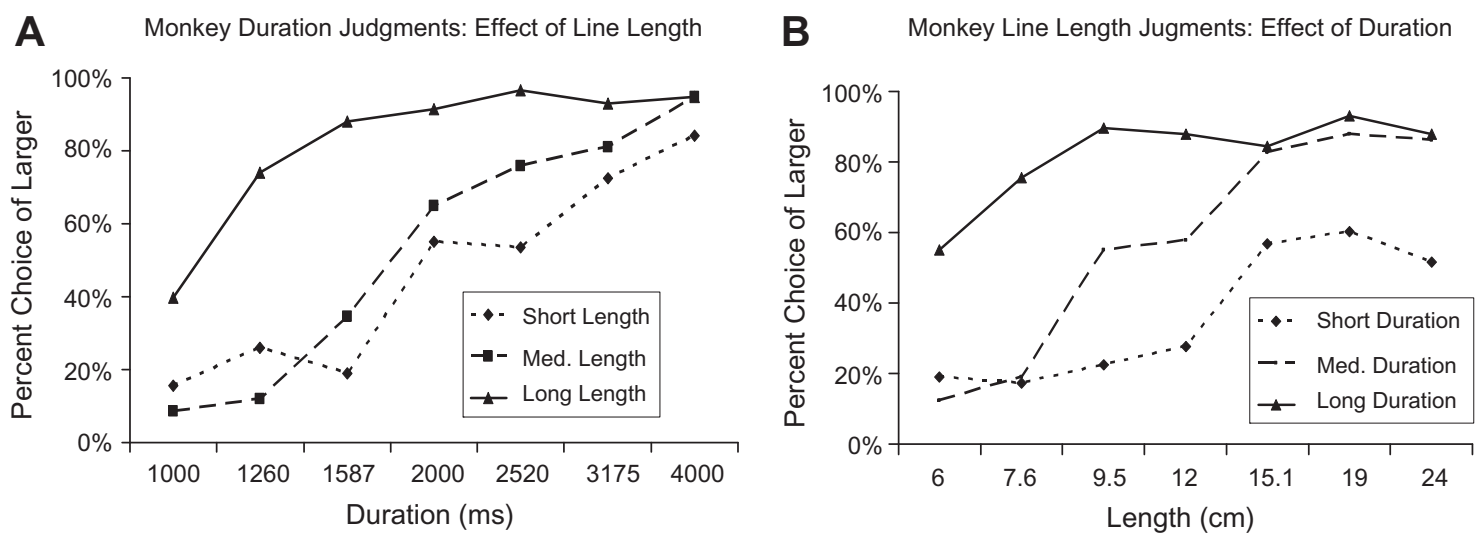

Fig. 6. (A) The bisection functions (in monkeys) for judgments of duration when crossed with short (6 cm), medium (12 cm), and long (24 cm) spatial lengths. (B) The bisection functions for judgments of spatial length when crossed with short (1000 ms), medium (2000 ms), and long (4000 ms) durations. When the line was shown, the background color of the screen remained light gray regardless of whether the monkeys were to judge line-length or duration.

length, PSE values were $15.9 \mathrm{~cm}, 9.96 \mathrm{~cm}, 5.8 \mathrm{~cm}$ for small, medium, and large duration values (Fig. 6B), $\chi^{2}(1, N=$ $1173)=164.83, p<0.001$, odds ratio $=3.32$, 95\% C.I. $2.71-$ 3.93. As in Experiment 2a, and in contrast to the pattern obtained with humans, time appears to have influenced space to a slightly larger degree than space influenced time $\chi^{2}(1, N=2347)=4.39, p=0.04$, odds ratio $=1.30,95 \%$ C.I. $0.97-1.63$. Importantly, however, the $95 \%$ C.I. around this odds ratio includes 1 (as it did in Experiment 2a), therefore we cannot conclude confidently that any cross-dimensional asymmetry exists.

Although the removal of the time and space judgment cues appears to have increased the size of the asymmetry slightly (pushing the $p$-value from marginally significant in Experiment 2a to significant in 2b), an analysis of the interaction between the size of the asymmetry and cue condition (cue vs. no cue) showed that the magnitude of bias created by the irrelevant dimension did not change; in fact, there was no interpretable difference in the size of the cross-dimensional asymmetry from Experiment $2 \mathrm{~A}$ to Experiment $2 \mathrm{~B}\left[\chi^{2}(1, N=4699)=0.002, p=0.96\right]$. Thus, forcing the monkeys to process both dimensions simultaneously did not change the pattern of influence between the two dimensions. This finding echoes earlier results in humans; the addition of pre-trial cues warning participants which dimension (space or time) would be relevant for a given judgment had no effect on the magnitude of the space-time asymmetry (compare Experiments 1 and 2 of Casasanto \& Boroditsky, 2008).

Finally, to compare the cross-dimensional interaction between humans and monkeys, we added species to the previous regression model, and looked at the three-way interaction between the irrelevant dimension, condition, and species. The comparison demonstrated that the asymmetrical pattern shown by humans was significantly different from the pattern shown by monkeys $\left[\chi^{2}(1, N=\right.$ $4884)=20.81, p<0.001$; odds ratio $=2.36$ ]. Thus, overall, the results are consistent with the idea that humans use space to structure representations of time, but this is unlikely to be the case for monkeys.

\section{General discussion}

Prior research has shown that space and time are related asymmetrically in the human mind. This has been demonstrated for both children and adults, using both linguistic and non-linguistic tasks (Boroditsky, 2000; Casasanto \& Boroditsky, 2008; Casasanto et al., 2010). This asymmetrical dependence is consistent with the idea that people use spatial schemas to think about time, as proposed by theories of metaphorical mental representation (e.g., Clark, 1973; Lakoff \& Johnson, 1980). Here we replicated the asymmetry between space and time in Englishspeaking adults using a nonverbal interval bisection task but we found a strikingly different pattern when the same task was performed by monkeys, for whom space and time appear to be symmetrically related.

In humans, the spatial extent of a stimulus biased judgments of its duration more than vice versa, as predicted by metaphor theory. People had a hard time ignoring irrelevant spatial information in the stimuli when performing a temporal bisection, but had an easier time ignoring irrelevant temporal information in the same stimuli when performing a spatial bisection. These data challenge ATOM, which implies a symmetric relationship between space and time (Walsh, 2003a). According to ATOM, space and time are simply two among the prothetic dimensions represented by a generalized mental magnitude system. At least $a$ priori, nothing in the theory suggests that representations in one dimension should depend asymmetrically on the other, contrary to our findings in humans.

Rhesus monkeys showed a different pattern from the humans on the same interval bisection task. In two experiments, irrelevant spatial information strongly affected time judgments, and irrelevant temporal information strongly affected space judgments. These bi-directional influences are best characterized as symmetric. Thus, the monkey data are consistent with predictions derived from ATOM, and inconsistent with metaphor theory.

In both of the monkey Experiments (2a and 2b) there was a trend toward an asymmetry in the opposite direction 
from the pattern seen in humans: time appeared to influence space slightly more than space influenced time. This pattern is not consistent with metaphor theory, nor with ATOM, nor with any other theoretical proposal we know of (cf. McGonigle \& Chalmers, 2001). Given that this trend was unpredicted, and also statistically unreliable, we will not attempt to interpret it here (i.e., for both experiments, the 95\% confidence intervals around the odds ratios estimating the magnitude of the cross-dimensional asymmetry included 1 ).

Could the behavioral asymmetry found in humans result from some asymmetry built into the stimuli, themselves? Is a line just an inherently spatial stimulus? At first glance, this may appear to be the case: intuitively, it may seem that space is an intrinsic property of a line presented on a screen, but time is not. However, upon closer inspection, this illusion dissolves. The fact that a line segment can be viewed at all demonstrates that it (like any object) exists in both space and time, and therefore has both intrinsic spatial and intrinsic temporal properties. The finding that monkeys do not show a space-time asymmetry supports the idea that the asymmetry between space and time is not inherent in the stimuli, themselves. Rather than being an intrinsic property of the stimuli, the space-time asymmetry we observe in humans participants is created in the mind of the human observer.

\subsection{Possible origins of space-time relationships}

Why are space and time interrelated so differently in the minds of humans and monkeys? Why do humans show a space-time asymmetry robustly, yet monkeys do not? One obvious possibility is that the difference between species lies in the availability of language. On some theories of metaphor, language plays a causal role in creating non-linguistic metaphorical mappings, encouraging language users to import the structure of concrete source domains like space into relatively abstract target domains like time, via analogy (e.g., Boroditsky, 2000; Gentner, 2001; Gentner \& Bowdle, 2008). Monkeys may not rely asymmetrically on space to represent time because they have no experience using the linguistic metaphors that would lead them to create asymmetric space-time mappings. This is possible, but it is not the only possible explanation for the cross-species differences we find.

Another possible source for these differences may be space-time correlations imposed by culture and technology. People, but not monkeys, are frequently exposed to spatial representations of time in artifacts like yearly calendars, progress bars on computers, battery indicators on cell phones, etc. Could these experiential differences create the cross-species patterns in space-time interactions that we observed? While it is likely that these cultural artifacts play a role, it is important to point out that humans and animals are exposed to many more fundamental spacetime correlations in their daily lives. Anytime something moves, or an organism ambulates through its environment, time and space are correlated, i.e. as moving object travels farther, more time passes. These certainly are not unique experiences created by progress bars, battery indicators, and other artificial media.
Second, we must ask ourselves why our culture so often uses spatial formats for keeping track of duration? It seems possible that progress bars, calendars, and other cultural conventions by which people spatially represent time in material artifacts are the result of the human propensity to spatialize time in our minds - not the cause. Clearly our experiments cannot disentangle these questions about causation.

On other theories of metaphor, cross-domain mappings can also arise in humans due to correlations in bodily experience, rather than through linguistic or cultural experience (e.g., Casasanto, 2008a; Lakoff \& Johnson, 1999). As evidence for this view, there are some non-linguistic mappings from source to target domains in humans' minds (i.e., mental metaphors) for which no corresponding verbal metaphors exist (Casasanto, 2008b, 2009a, 2009b). For example, across cultures, right-handers tend to associate good things with the right side of space and bad things with the left, but left-handers show the opposite pattern, implicitly associating good with left and bad with right (Casasanto, 2009b; Casasanto \& Jasmin, 2010). The righthanders' pattern is consistent with idioms in language and culture that link right with good (e.g., the right answer, swearing to tell the truth with the right hand), but the lefthanders' pattern goes the opposite direction. This pattern could not develop due to language or enculturation, but appears to result from implicit associations between two kinds of bodily experience: motoric and emotional. People come to associate good things with the side of space on which they can act more fluently with their dominant hand, and bad things with the side on which they act more clumsily (Casasanto, 2009b). The implicit 'left is good' mapping in left-handers demonstrates that at least some mental metaphors can develop independent of any corresponding linguistic metaphors.

Arguably, mental metaphors from space to time could also develop due to non-linguistic interactions between body and world. For example, our interactions with moving objects could teach us to associate distance with time since, invariably, as things travel farther more time passes. Knowing this kind of space-time correlation is useful for understanding and acting upon the physical world in various ways, from swatting a fly to planning a cross-country trip. Correlation is a symmetric relationship, but we may rely asymmetrically on the dimension that is easier to perceive, remember, or reconstruct from physical evidence. That is, we may use space heuristically as an index of time because, in many cases, the spatial dimension of an event is more durable and more perceptually available than the temporal (Casasanto, 2009a; Casasanto \& Boroditsky, 2008; Casasanto et al., 2010). Like other heuristics based on regularities in our environment, assuming that longer in space corresponds to longer in time is generally useful for judging natural space-time relationships, but can lead to judgment errors in laboratory tasks that are constructed to expose this implicit 'rule of thumb' (i.e., here we varied distance and time orthogonally, disrupting the natural positive correlation between these dimensions).

Thus, mental metaphors linking space and time could develop in humans due either to correlations in linguistic/cultural experience or to correlations in bodily experi- 
ence with the physical world - or due to some combination of linguistic, cultural, and bodily experience. The results of the monkey experiments leave all of these possibilities open. If the monkeys had shown a similar space-time asymmetry to humans, this would have provided an existence proof that mental metaphors can develop in a mind without language. But importantly, the fact that monkeys showed a different pattern from humans does not license the opposite conclusion; we cannot conclude that language is necessary for the development of mental metaphors from space to time.

It is possible that space-time relationships in monkeys and humans differed because of language-related differences, alternatively they may differ as a result of any of the myriad of bodily differences between species that constrain the way they interact with the physical environment. For example, humans are typically restricted to moving in two dimensions while rhesus monkeys readily move in three dimensions. Humans are also capable of throwing objects, whereas rhesus monkeys are not (Wood, Glynn, \& Hauser, 2007; see Casasanto (2009a) for further discussion). These physical differences between the two species may yield differences in how humans and monkeys experience space-time relationships. To the extent that mental metaphors from space to time depend on such physical interactions, the cross-species difference we observed should be expected - even if we set aside the possible role of language in creating mental metaphors.

\subsection{Cross-species similarities and differences}

The strong cross-species difference we report here stands in contrast to numerous studies showing commonalities in the way humans and animals represent analog magnitudes in basic prothetic dimensions (Allan \& Gibbon, 1991; Cantlon \& Brannon, 2006; Meck \& Church, 1983). In particular, a rich literature shows that animals and humans share many qualitative similarities in timing behavior. In temporal bisection tasks, both animals and humans show PSE values at the geometric mean (Allan \& Gibbon, 1991; Church \& Deluty, 1977), and further, both animals and humans produce discrete categorical long-short boundaries in temporal bisection tasks when feedback on the anchors is sparse (Penney, Gibbon, \& Meck, 2008). In tasks where time intervals are compared, both humans and animals show accuracy functions that follow Weber's law, whereby the ratio of the intervals determine how easy they are to discriminate (e.g., Church \& Gibbon, 1982; Lejeune \& Wearden, 2006; Wearden, 1992). Weber's law is also observed in tasks where animals and humans reproduce specific time intervals, with the standard deviation of the estimates increasing linearly with the length of the temporal interval (e.g., Higa \& Simm, 2004; Rakitin et al., 1998; Roberts, Cheng, \& Cohen, 1989).

Based on this evidence, it appears that animals and humans share common mechanisms that underlie their interval timing abilities (see Malapani \& Fairhurst, 2002). Although these similarities are compelling, it is important to note that humans have a much richer conceptualization of time that goes far beyond the types of timing abilities shown thus far in animals. Humans can think about time periods that are impossible to actually experience, either because they are too vast (e.g., ice ages, millennia), because they happened in the past (the Age of Enlightenment), or because they have yet to occur (the 22nd century). We can think about relationships between hypothetical past events (e.g. I could have gone to the store before going to work) or future events (e.g. I could leave the Wednesday after next). We can even entertain temporal scenarios that could never possibly occur, as when we imagine journeying back in time to an earlier era.

Thinking about relationships between events in the past and future may require different types of representations from those used for discriminating, matching, or reproducing temporal intervals, but these, too, may be dependent on space. It has been suggested previously that our heuristic use of space for representing basic temporal sequences and intervals may have paved the way for more sophisticated and abstract kinds of temporal reasoning to emerge in humans. Spatial paths can be traveled forward or backward. Once time is conceptualized as a spatial path, then time can be traveled forward or backward - at least in our imaginations (Casasanto \& Boroditsky, 2008; Casasanto et al., 2010).

\subsection{Spatial metaphor and mental time travel}

If spatializing time enables humans to conceptualize the remote past or the distant future, could the fact that monkeys do not spatialize time the way we do prevent them from thinking about their past and future? Reasoning about the future is closely tied to the ability to form episodic memories (Spreng \& Levine, 2006; Suddendorf \& Corballis, 2007). Episodic memory is explained as a type of mental time travel, in which past events are mentally reexperienced and localized in space and time (Tulving, $1972,1985)$, a capacity that has been argued to be uniquely human. Because it is impossible to know whether an animal can mentally re-experience a past event, research has focused on whether animals can understand events in terms of what, where, and when (www) (Clayton, Salwiczek, \& Dickinson, 2007).

Research investigating the www question in rhesus monkeys is sparse, but some evidence suggests that rhesus monkeys can meet the www criterion in short-term memory tasks. Hoffman, Beran, and Washburn (2009) found that, in a delayed matching to sample task, rhesus monkeys could selectively determine the identity (what), spatial location (where), and time of presentation (when) of a particular stimulus. However, in long-term memory tasks, the evidence is less clear. Hampton, Hampstead, and Murray (2005) found that rhesus monkeys could remember the locations of preferred food items hidden in a room, but the monkeys were unable to remember when they acquired that knowledge. This suggests that rhesus monkeys may have difficulty forming long-term episodiclike memories.

Other animals, however, have proven more successful in meeting the www criterion (Babb \& Crystal, 2006; Clayton \& Dickinson, 1998; Clayton \& Dickinson, 1999; Dere, Huston, \& De Souza Silva, 2005; Zhou \& Crystal, 2009). Clayton and Dickinson (1998) found that scrub jays 
remember the type and location of previously cached food, and they also use their knowledge of when the food was hidden in order to make decisions about which caches to visit. Further, studies show that scrub jays plan for the future when caching their food (e.g., Correia, Dickinson, \& Clayton, 2007; Raby, Alexis, Dickinson, and Clayton, 2007). Other studies suggest that great apes may 'think ahead' under some circumstances, as well, choosing tools that will be useful for solving particular problems in the future (Mulcahy \& Call, 2006; Osvath, 2009).

Overall, the evidence for mental time travel in animals remains controversial. In a review of the available data, Suddendorf and Corballis (2007) concluded that studies "have not provided sufficient grounds to reverse existing skepticism about the existence of mental time travel in non-human animals" (p. e2). For example, some have questioned whether scrub jays' remarkable abilities represent a general capacity to think about and plan for the future, or whether they represent domain-specific learning algorithms related to food caching (Suddendorf, 2006; Suddendorf \& Corballis, 2007).

Although humans' capacity for mental time travel appears to be the most advanced, some non-human animals may be able to reason about the past and future more effectively than others. It is a question for future comparative research whether animals that exhibit a greater capacity for mental time travel also show a greater dependence of time on space in their more basic temporal representations. Such a finding would suggest an unexplored link between the human capacity to spatialize time and our ability to form episodic memories.

\section{Conclusions}

Tests of how space and time are related in the minds of humans and monkeys revealed a clear cross-species difference. In humans, space and time were asymmetrically related. Spatial information influenced temporal judgments much more than temporal information influenced spatial judgments, consistent with previous findings in human adults and children. In monkeys, however, space and time were symmetrically related. Spatial information strongly influenced temporal judgments, and temporal information strongly influenced spatial judgments.

The asymmetric relationship we find in humans supports theories of metaphorical mental representation, according to which abstract domains like time are structured, in part, by mappings from more concrete domains like space. By contrast, the symmetric relationship in monkeys is most consistent with ATOM, which suggests that space and time are represented by a common metric for analog magnitudes. Together, these data raise the possibility that the capacity to represent abstract magnitudes metaphorically may be uniquely human.

\section{Acknowledgements}

We thank Monica Carlson, Evan MacLean, and Sayed Zaman for assisting with this research. We also thank the members of the Brannon lab for their helpful discussion of these data. This work was supported by an NICHD RO1 (HD49912), an NSF Career award Grant No. 0448250, a James McDonnell scholar award to Elizabeth M. Brannon, and by National Institutes of Health under Ruth L. Kirschstein National Research Service Award No. F32MH082623 to Dustin J. Merritt.

\section{References}

Allan, L. G., \& Gibbon, J. (1991). Human bisection at the geometric mean. Learning and Motivation, 22, 39-58.

Babb, S. J., \& Crystal, J. D. (2006). Episodic-like memory in the rat. Current Biology, 16(13), 1317-1321.

Basso, G., Nichelli, P., Frassinetti, F., \& diPellegrino, G. (1996). Time perception in a neglected space. NeuroReport, 7(13), 2111-2114.

Boroditsky, L. (2000). Metaphoric structuring: Understanding time through spatial metaphors. Cognition, 75(1), 1-28.

Bottini, R., \& Casasanto, D. (2010). Implicit spatial length modulates time estimates, but not vice versa. In C. Hölscher et al. (Eds.). Spatial cognition (Vol. VII, pp. 152-162). New York: Springer.

Cantlon, J. F., \& Brannon, E. M. (2006). Shared system for ordering small and large numbers in monkeys and humans. Psychological Science, 17(5), 402-407.

Cantlon, J. F., Platt, M. L., \& Brannon, E. M. (2009). Beyond the number domain. Trends in Cognitive Science, 13(2), 83-91.

Casasanto, D. (2008a). Who's afraid of the Big Bad Whorf? Cross-linguistic differences in temporal language and thought. Language Learning, $58(1), 63-79$.

Casasanto, D. (2008b). Similarity and proximity: When does close in space mean close in mind? Memory $\mathcal{E}$ Cognition, 36(6), 1047-1056.

Casasanto, D. (2009a). When is a linguistic metaphor a conceptual metaphor? In V. Evans \& S. Pourcel (Eds.), New directions in cognitive linguistics (pp. 127-145). Amsterdam: John Benjamins.

Casasanto, D. (2009b). Embodiment of abstract concepts: Good and bad in right- and left-handers. Journal of Experimental Psychology: General, 138(3), 351-367.

Casasanto, D., \& Boroditsky, L. (2008). Time in the mind: Using space to think about time. Cognition, 106(2), 579-593.

Casasanto, D., Fotakopoulou, O., \& Boroditsky, L. (2010). Space and time in the child's mind: Evidence for a cross-dimensional asymmetry. Cognitive Science, 34, 387-405.

Casasanto, D., \& Jasmin, K. (2010). Good and bad in the hands of politicians: Spontaneous gestures during positive and negative speech. PLoS ONE, 5(7), e11805.

Church, R. M., \& Deluty, M. Z. (1977). Bisection of temporal intervals. Journal of Experimental Psychology: Animal Behavior Processes, 3(3), 216-228.

Church, R. M., \& Gibbon, J. (1982). Temporal generalization. Journal of Experimental Psychology - Animal Behavior Processes, 8(2), 165-186.

Clark, H. H. (1973). Space, time, semantics and the child. In T. E. Moore (Ed.), Cognitive development and the acquisition of language (pp. 27-63). New York: Academic Press.

Clayton, N. S., \& Dickinson, A. (1998). Episodic-like memory during cache recovery by scrub jays. Nature, 395(6699), 272-274.

Clayton, N. S., \& Dickinson, A. (1999). Scrub jays (Aphelocoma coerulescens) remember the relative time of caching as well as the location and content of their caches. Journal of Comparative Psychology, 113(4), 403-416.

Clayton, N. S., Salwiczek, L. H., \& Dickinson, A. (2007). Episodic memory. Current Biology, 17(6), R189-191.

Correia, S. P., Dickinson, A., \& Clayton, N. S. (2007). Western scrub-jays anticipate future needs independently of their current motivational state. Current Biology, 17(10), 856-861.

Dehaene, S., Bossini, S., \& Giraux, P. (1993). The mental representation of parity and number magnitude. Journal of Experimental Psychology General, 122(3), 371-396.

DeLong, A. J. (1981). Phenomenological space-time: Toward an experiential relativity. Science, 213(4508), 681-683.

Dere, E., Huston, J. P., \& De Souza Silva, M. A. (2005). Episodic-like memory in mice: Simultaneous assessment of object, place and temporal order memory. Brain Research Protocols, 16(1-3), 10-19.

Feigenson, L. R. (2007). The equality of quantity. Trends in Cognitive Sciences, 11(5), 185-187.

Gallistel, C., \& Gelman, R. (2000). Non-verbal numerical cognition: From reals to integers. Trends in Cognitive Sciences, 4(2), 59-65. 
Gentner, D. (2001). Spatial metaphors in temporal reasoning. In M. Gattis (Ed.), Spatial schemas in abstract thought (pp. 203-222). Cambridge, MA: MIT Press.

Gentner, D., \& Bowdle, B. (2008). Metaphor as structure-mapping. In R. Gibbs (Ed.), The Cambridge handbook of metaphor and thought (pp. 109-128). New York, NY: Cambridge University Press.

Hampton, R. R., Hampstead, B. M., \& Murray, E. A. (2005). Rhesus monkeys (Macaca mulatta) demonstrate robust memory for what and where, but not when, in an open-field test of memory. Learning and Motivation, 36, 245-259.

Higa, J. J., \& Simm, L. A. (2004). Interval timing in Siamese fighting fish (Betta splendens). Behavioural Processes, 67(3), 501-509.

Hoffman, M. L., Beran, M. J., \& Washburn, D. A. (2009). Memory for "what", "where", and "when" information in rhesus monkeys (Macaca mulatta). Journal of Experimental Psychology: Animal Behavior Processes, 35(2), 143-152.

Hubbard, E. M., Piazza, M., Pinel, P., \& Dehaene, S. (2005). Interaction between number and space in parietal cortex. Nature Reviews Neuroscience, 6(6), 435-448.

Lakoff, G., \& Johnson, M. (1980). Metaphors we live by. Chicago: University of Chicago Press.

Lakoff, G., \& Johnson, M. (1999). Philosophy In the flesh: The embodied mind and its challenge to western thought. Basic books.

Lejeune, H., \& Wearden, J. H. (2006). Scalar properties in animal timing Conformity and violations. Quarterly Journal of Experimental Psychology, 59(11), 1875-1908.

Leon, M. I., \& Shadlen, M. N. (2003). Representation of time by neurons in the posterior parietal cortex of the macaque. Neuron, 38(2), 317-327.

Malapani, C., \& Fairhurst, S. (2002). Scalar timing in animals and humans. Learning and Motivation, 33, 156-176.

McGonigle, B., \& Chalmers, M. (2001). Spatial representation as cause and effect: Circular causality comes to cognition. In M. Gattis (Ed.), Spatial schemas and abstract thought. London: MIT Press.

Meck, W. H., \& Church, R. M. (1983). A mode control model of counting and timing processes. Journal of Experimental Psychology - Animal Behavior Processes, 9(3), 320-334.

Mulcahy, N. J., \& Call, J. (2006). Apes save tools for future use. Science, 312(5776), 1038-1040.

Murphy, G. L. (1996). On metaphoric representation. Cognition, 60(2), 173-204.

Murphy, G. L. (1997). Reasons to doubt the present evidence for metaphoric representation. Cognition, 62(1), 99-108.

Nieder, A., Freedman, D. J., \& Miller, E. K. (2002). Representation of the quantity of visual items in the primate prefrontal cortex. Science, 297(5587), 1708-1711.

Oliveri, M., Vicario, C. M., Salerno, S., Koch, G., Turriziani, P., Mangano, R., et al. (2008). Perceiving numbers alters time perception. Neuroscience Letters, 438(3), 308-311.

Onoe, H., Komori, M., Onoe, K., Takechi, H., Tsukada, H., \& Watanabe, Y. (2001). Cortical networks recruited for time perception: A monkey positron emission tomography (pet) study. Neuroimage, 13(1), 37-45.

Osvath, M. (2009). Spontaneous planning for future stone throwing by a male chimpanzee. Current Biology, 19(5), R190-191.

Penney, T. B., Gibbon, J., \& Meck, W. H. (2008). Categorical scaling of duration bisection in pigeons (Columba livia), mice (Mus musculus), and humans (Homo sapiens). Psychological Science, 19(11), 1103-1109.
Piaget, J. (1927). The child's conception of time. New York: Ballantine Books. Raby, C. R., Alexis, D. M., Dickinson, A., \& Clayton, N. S. (2007). Planning for the future by western scrub-jays. Nature, 445(7130), 919-921.

Rakitin, B. C., Gibbon, J., Penney, T. B., Malapani, C., Hinton, S. C., \& Meck, W. H. (1998). Scalar expectancy theory and peak-interval timing in humans. Journal of Experimental Psychology: Animal Behavior Processes, 24(1), 15-33.

Roberts, W. A., Cheng, K., \& Cohen, J. S. (1989). Timing light and tone signals in pigeons. Journal of Experimental Psychology: Animal Behavior Processes, 15(1), 23-35.

Sarrazin, J. C., Giraudo, M. D., Pailhous, J., \& Bootsma, R. J. (2004). Dynamics of balancing space and time in memory: Tau and kappa effects revisited. Journal of Experimental Psychology: Human Perception and Performance, 30(3), 411-430.

Spreng, R. N., \& Levine, B. (2006). The temporal distribution of past and future autobiographical events across the lifespan. Memory $\mathcal{E}$ Cognition, 34(8), 1644-1651.

Suddendorf, T. (2006). Behavior. Foresight and evolution of the human mind. Science, 312(5776), 1006-1007.

Suddendorf, T., \& Corballis, M. C. (2007). The evolution of foresight: What is mental time travel, and is it unique to humans? Behavioral and Brain Sciences, 30(3), 299-313 (discussion 313-251).

Sawamura, H., Shima, K., \& Tanji, J. (2002). Numerical representation for action in the parietal cortex of the monkey. Nature, 415(6874), 918-922.

Srinivasan, M., \& Carey, C. (2010). The long and short of it: On the nature and origin of functional overlap between representations of space and time. Cognition, 116, 217-241.

Stein, J. F. (1989). Representation of egocentric space in the posterior parietal cortex. Quarterly Journal of Experimental Physiology, 74(5), 583-606.

Tulving, E. (1985). Memory and consciousness. Canadian Psychology/ Psychologie Canadienne, 26, 1-12.

Tulving, E. (Ed.). (1972). Episodic and semantic memory. New York: Academic Press.

Vicario, C. M., Pecoraro, P., Turriziani, P., Koch, G., Caltagirone, C., \& Oliveri, M. (2008). Relativistic compression and expansion of experiential time in the left and right space. PLoS One, 3(3), e1716.

Walsh, V. (2003a). A theory of magnitude: Common cortical metrics of time, space and quantity. Trends in Cognitive Sciences, 7(11), 483-488.

Walsh, V. (2003b). Cognitive neuroscience. Numerate neurons. Current Biology, 13(11), R447-448.

Wearden, J. H. (1992). Temporal generalization in humans. Journal of Experimental Psychology: Animal Behavior Processes, 18(2), 134-144.

Wilson, F. A., Scalaidhe, S. P., \& Goldman-Rakic, P. S. (1993). Dissociation of object and spatial processing domains in primate prefrontal cortex. Science, 260(5116), 1955-1958.

Wood, J. N., Glynn, D. D., \& Hauser, M. D. (2007). The uniquely human capacity to throw evolved from a non-throwing primate: An evolutionary dissociation between action and perception. Biology Letters, 3(4), 360-364.

Zhou, W., \& Crystal, J. D. (2009). Evidence for remembering when events occurred in a rodent model of episodic memory. Proceedings of the National Academy of Sciences USA, 106(23), 9525-9529. 